\title{
Equations of State in Stellar Structure and Evolution
}

\section{H. M. VAN HORN}

Department of Physics and Astronomy, C. E. Kenneth Mees Observatory, and Laboratory for Laser Energetics, University of Rochester, Rochester, NY 14627-0011. Current address: Division of Astronomical Sciences, Room 1015, National Science Foundation, 4201 Wilson Boulevard, Arlington, VA 22290

\begin{abstract}
In this paper I summarize some of the recent advances in studies of dense matter. Research on phase separation in the binary ionic mixtures (BIMs) that constitute the matter in white dwarfs has been motivated by the need to obtain accurate estimates for the ages of the faintest white dwarfs and thus of the disk of our Galaxy. Substantial age increases appear possible, but it is not yet clear whether such large increases occur in real white dwarfs. A second advance is the prediction, based on state-of-the-art physical calculations, that ionization of $\mathrm{H}$ at low temperatures and increasing densities may occur via a first-order "plasma phase transition" (PPT). Astrophysical consequences of this result are still being explored in an effort to test this prediction. Related to these equation-of-state calculations are calculations of the enhancement of nuclear reaction rates at high densities. New thermonuclear rates have been computed for $\mathrm{C}+\mathrm{C}$ reactions in BIMs, although there is currently some controversy about results at the highest densities. New pycnonuclear reaction rates have also been calculated for BIMs, and it has been suggested that He-burning at $T=0$ may occur through a first-order phase transition. Finally, calculations of the equation of state of matter in strong magnetic fields and of radiative opacities at high densities have undergone very recent and substantial improvements, which are just beginning to be utilized in astrophysical calculations.
\end{abstract}

Dans cette revue, je résumme les travaux les plus récents dans l'étude 
de la matière dense. Les recherches sur la séparation de phase dans les mélanges ioniques binaires qui constituent l'intérieur des naines blanches ont été motivées par la nécessité d'obtenir une estimation correcte de l'age des naines blanches les moins brillantes et donc du disque de notre Galaxie. Il apparait possible d'accroitre l'age substantiellement, mais il n'est pas encore certain qu'un tel accroissement ait réellement lieu dsans les naines blanches. Une seconde percée est la prédiction, basée sur des calculs trò détaillés, que l'ionisation de $\mathrm{H}$ a basse température et haute pression ait lieu au travers d'une "transition de phase plasma (PPT)". Les conséquences astrophysiques de ce résultat sont actuellement encore en cours d'étude, afin de tester cette prédiction. En relation avec ces équations d'état, ont lieu des calculs d'accroissement de taux de réactions nucléaires à haute densité. De nouveaux taux termonucléaires ont été calculés pour la réaction $\mathrm{C}+\mathrm{C}$ dans les mélanges binaires, bien au'il y ait actuellement une controverse quant à ces résultats. De nouveaux taux pycnonucléaires ont été calculés pour les mélanges, et il a été suggéré que le brûlage de $\mathrm{He}$ a $T=0$ ait lieu au travers d'une transition de phase du premier ordre. Enfin, des calculs de l'équation d'état de la matière dans les champs magnétiques forts et des opacités radiatives à haute densité ont été effectués récemment.

\subsection{Introduction}

The purpose of this paper is to summarize various problems related to the equations of state (EOS) used in stellar structure and evolution calculations. I shall be concerned with quantities such as the EOS itself, various transport coefficients, nuclear reaction rates, and so forth. I shall endeavor to summarize some of the most recent advances in these areas, but it is not possible here to cover all topics of interest. In particular, there have been some interesting recent calculations of the EOS of matter in strong magnetic fields which are mentioned only briefly at the end of this paper.

Let me begin with a reminder of the parameters needed for stellar evolution calculations. The four equations describing the time-dependent structure of a star are (cf. Clayton 1968, pp. $436 \mathrm{ff}$.):

$$
\begin{aligned}
& \frac{d M_{r}}{d r}=4 \pi r^{2} \rho, \\
& \frac{d P}{d r}=-\rho \frac{G M_{r}}{r^{2}}, \\
& \frac{d T}{d r}=-\frac{3}{4 a c} \frac{\kappa \rho}{T^{3}} \frac{L_{r}}{4 \pi r^{2}},
\end{aligned}
$$


and

$$
\frac{d L_{r}}{d r}=4 \pi r^{2} \rho\left(\varepsilon-T \frac{\partial S}{\partial t}\right)
$$

In order to solve these equations, we need to know the following properties of stellar matter: the pressure $P=P(\rho, T)$, the entropy $S=S(\rho, T)$, the opacity $\kappa=\kappa(\rho, T)$, and the net nuclear energy generation rate $\varepsilon=\varepsilon(\rho, T)$. The relations giving the dependence of the pressure and entropy upon the thermodynamic state variables $\rho$, the mass density, and $T$, the temperature, are collectively called the "equation of state." Quantities such as the radiative opacity $\kappa$ and the so-called "conductive opacity" $\kappa_{c}$, proportional to the inverse of the thermal conductivity, are termed transport coefficients; they determine the rate of transport of some physical quantity, such as heat. For some purposes, we also need to know the viscosity (which controls the rate of transport of momentum) or the ambipolar diffusion coefficients (which govern the rate of gravitational separation of elements in a star); for the most part I shall neglect these quantities in this brief review. The thermonuclear and pycnonuclear (density-induced) reaction rates $\varepsilon$ and the neutrino loss rates $\varepsilon_{\nu}$ round out the list of physical quantities needed.

It is also useful to have some idea of the values of the dimensionless physical parameters that characterize the state of matter at different temperatures and densities. Van Horn (1991) has illustrated this for plasmas composed of $\mathrm{H}$ or of Fe. Here we briefly describe the changes in the physical conditions, beginning at low $\rho$ and at thermal energies $k T$ much less than the ionization potentials of the atoms. Under these conditions, matter consists of an ideal gas of neutral atoms or molecules. As the temperature increases, but still at low densities, the molecules first dissociate into neutral atoms, and the atoms subsequently ionize. At sufficiently high $T$, matter consists of a fully ionized plasma of bare atomic nuclei and electrons. Under these conditions, matter can still be described as a mixture of ideal gases (the ions and electrons). If the density is now increased along some isotherm, new, density-dependent effects begin to appear. As the electrons in the plasma become crowded increasingly close together, degeneracy first becomes important. This occurs when $k T \sim \epsilon_{F}$, where $\epsilon_{F}$ is the Fermi energy. At still higher densities, the Coulomb interaction energy $(Z e)^{2} / a$ between the electrons and ions becomes comparable to $k T$. Here $a$ is a measure of the average separation of electrons and ions; it is of the order of the size of the "Wigner-Seitz sphere" containing just enough electrons to neutralize the ionic charge. The quantity $\Gamma \equiv(Z e)^{2} / a k T$ is the dimension- 
less parameter generally used to characterize the strength of the Coulomb interaction.

The condition $\Gamma<1$ corresponds to a high-temperature, low-density, fully ionized plasma. If we continue our tour through the $(\rho, T)$ plane along an isochore at very high density but decreasing temperature, the parameter $\Gamma$ continually increases. This corresponds to the increasing importance of the Coulomb interactions. At sufficiently low $T$, coresponding to $\Gamma \approx 180$, the plasma actually freezes into a crystalline lattice structure. This was first discovered more than 25 years ago as a result of numerical simulations of the properties of the idealized "one-component plasma" (OCP), consisting of a single species of ions in a uniform neutralizing background. Advances in computer technology have now permitted Monte Carlo simulations extending over tens or hundreds of millions of configurations for thousands of particles of several different species and have greatly improved the precision of our knowledge of the thermodynamic properties of very dense plasmas. Much of the discussion in the remainder of this paper concerns results we have learned from such investigations. Indeed, for "binary ionic mixtures" (BIMs), consisting of two species of ions in a uniform background, much of the recent discussion has focused on calculations of the phase diagrams of the mixtures and the investigation of the possible astrophysical consequences of phase separation.

In $\$ 2$ below, I first discuss the implications of the phase diagrams of various mixtures for the ages of the coolest white dwarf stars. Next, in $§ 3, I$ consider the so-called "plasma phase transition," which has been suggested as the process by which the transition from low-density, molecular $\mathrm{H}_{2}$ to high-density metallic hydrogen occurs. I describe some recent work on nuclear reaction rates at high densities in $\$ 4$, and in $\$ 5$ I conclude with a list of some important problems that still need attention.

\subsection{EOS I: Phase Separation in White Dwarfs and the Age of the Galaxy}

Much of the reason for the current interest in the problem of phase separation in white dwarfs stems from the prospect of using white dwarf cooling theory to measure the age of the disk of our Galaxy. This method is based on the observed shortfall in the white dwarf luminosity function at $\log L / L_{\odot} \approx-4.5$ (cf. Liebert, Dahn, and Monet 1988); the "luminosity function" is effectively the number density of stars per unit interval of $\log L / L_{\odot}$. Using the best existing white dwarf cooling calculations, the ages of the faintest white dwarfs are found to be $\sim 9 \mathrm{Gyr}$ (Winget et al. 1987; 
Iben and Laughlin 1989). This result is surprising, because it is much less than the ages of the oldest globular cluster stars, which lie in the range from $\sim 12$ to $18 \mathrm{Gyr}$ (cf. Norris and Green 1989). This disagreement has important astrophysical consequences, because it implies either (1) that the ages of the white dwarfs are wrong because of unanticipated effects that lengthen the white dwarf cooling times or (2) that we require a new paradigm for galaxy formation.

The possibility that a new model for galaxy formation may be needed is the more interesting one, as it would have profound implications for many different areas of astrophysics. There are even some indications that this may be the case (Blitz 1993). For example, Larson's early theoretical calculations of pressure-supported collapse (Larson 1976, cited by Norris and Green 1989) indicate that the disk of our Milky Way Galaxy may have formed from the inside out, with a lapse of several billion years between the beginning of star formation in the central bulge and the onset of star formation out at the $\sim 10 \mathrm{kpc}$ distance of the Sun from the galactic center. In addition, some observations (Sarajedini and King 1989, Lee 1992, van den Bergh 1993) appear to indicate a real spread in the ages of the globular clusters. If this is the case, it supports a gradual collapse model for the formation of the Galaxy.

However, before one can uncritically accept the idea that a new model for galaxy formation is demanded by the evidence, it is important to investigate the alternative. In particular, are there additional energy sources that can "turn on" late in the evolution of a white dwarf and prolong the cooling times? One possibility that has been suggested to do precisely this is phase separation. The idea is that as a white dwarf cools to the point at which its center begins to freeze, it may undergo phase separation into, e.g., a less dense fluid and a denser solid. If this occurs, the solid can "snow out," falling toward the center of the white dwarf and liberating gravitational potential energy. If the density contrast is sufficiently great, the energy liberated can be substantial and may prolong the white dwarf cooling times significantly. It should also be noted, however, that this additional energy release produces a bump in the white dwarf luminosity function at low luminosities (Garcia-Berro et al. 1988); the prolongation of the cooling times means that more white dwarfs "pile up" in the lowest luminosity intervals. This effect is not seen in the observational luminosity function. However, the error bars are so large at the critical, low-luminosity end of the range that this effect might not have been detected, even if if it is present.

White dwarfs are thought to consist mainly of $C$ and $O$, and Stevenson (1980) originally suggested that the phase diagram of the $\mathrm{C} / \mathrm{O}$ mixture 
might have either a eutectic or a spindle form. If the phase diagram were of the eutectic type, the density contrast between the two phases would be substantial, and a considerable prolongation of the cooling times would occur. Conversely, if the phase diagram were of the spindle type, the density contrast would be much smaller, and a correspondingly smaller age change would result.

The importance of this issue led two groups independently to investigate this question using the best modern calculations. Barrat, Hansen, and Mochkovitch (1988) performed a density-functional calculation and found a spindle-shaped phase diagram. Ichimaru, Iyetomi, and Ogata (1988) carried out an analysis based on their very high-precision Monte Carlo calculations and found an azeotropic phase diagram (that is, a phase diagram that resembles a spindle shape except at low $\mathrm{O}$ abundances). In both cases, very little density contrast was produced upon phase separation, and the age-extension inferred for the oldest white dwarfs was found to be only $\Delta t \sim 0.5 \mathrm{Gyr}$ ).

Another possibility that has been considered is $\mathrm{C} / \mathrm{Ne}$ phase separation (Garcia-Berro et al. 1988). Although the cosmic abundance of $\mathrm{Ne}$ is too small to produce a significant effect, the $\mathrm{Ne}$ abundance in white dwarfs is thought to be produced by nucleosynthesis from the primordial CNO elements during the prior stages of evolution and to be considerably larger than the cosmic abundance. The CNO elements incorporated into stars at birth are all converted primarily to ${ }^{14} \mathrm{~N}$ during H-burning on the main sequence. The ${ }^{14} \mathrm{~N}$ is subsequently converted into ${ }^{22} \mathrm{Ne}$ during He-burning in the red-giant phase, with an abundance as large as $X_{\mathrm{Ne}} \sim 0.02$. This can in principle lead to significant energy release associated with $\mathrm{C} / \mathrm{Ne}$ phase separation late in the cooling of a white dwarf. Preliminary estimates by Garcia-Berro et al. gave varying age-extensions $\Delta t$ ranging up to several Gyr, depending upon the $\mathrm{C} / \mathrm{O}$ ratio.

To investigate further the effects of phase separation involving trace elements, Segretain and Chabrier (1993) and Ogata et al. (1993) have recently completed independent calculations of the phase diagrams of binary ionic mixtures (BIMs) involving various trace species. Segretain and Chabrier employed density-functional-based calculations, while Ogata et al. employed high-precision Monte Carlo calculations. Ogata et al. (1993) found spindle-shaped and azeotropic phase diagrams for low values of the charge ratio $Z_{2} / Z_{1}$, such as the values appropriate for $0 / \mathrm{Ne}$ or $\mathrm{C} / \mathrm{O} \mathrm{BIMs}$, while the phase diagram assumes a eutectic form for larger values of $Z_{2} / Z_{1}$, as appropriate for C/Ne or C/Fe BIMs. Segretain and Chabrier (1993) obtained results that differ in detail but which are qualitatively very similar 
to those of Ogata et al. This similiarity provides considerable confidence that the general features of both sets of results are reliable.

Ogata (1993) has recently performed the first detailed stellar evolution calculations that include $\mathrm{C} / \mathrm{Ne}$ phase separation self-consistently. His computations show that the age of a $0.6 M_{\odot} \mathrm{C}$ white dwarf with $X_{\mathrm{Ne}}=0.02$ at $\log L / L_{\odot}=-4.5$ increases by $5.7 \mathrm{Gyr}$ when $\mathrm{C} / \mathrm{Ne}$ phase separation is included. If it is not washed out by other effects, this is large enough to resolve much of the discrepancy between the white dwarf ages and those of the globular cluster stars. However, the primordial CNO abundances are considerably less than $X_{\mathrm{Ne}}=0.02$, and since the age-extension $\Delta t$ scales approximately linearly with these abundances it is not yet clear that the ages of the coolest white dwarfs actually are substantially increased. In addition, while Ogata and his collaborators have not yet computed the white dwarf luminosity function associated with their evolutionary calculations, from rather general considerations one expects a substantial bump. Averaging over the pre-white-dwarf mass distribution may flatten this bump somewhat, but these computations remain to be completed.

In two recent papers, Segretain et al. (1994) and Hernanz et al. (1994) carefully examined the effect of the different crystallization processes on the cooling time and on the luminosity function of white dwarfs. Their calculation of the luminosity function takes into account the white dwarf mass distribution function and a model for the chemical evolution of the Galaxy. These authors found out that the crystallization of trace elements alter severely the cooling time of the star, but do not modify significantly the position of the cut-off in the luminosity function, a direct consequence of the strong dependence of metallicity upon galactic evolution.

\subsection{EOS II: Dense Hydrogen in Brown Dwarfs and Giant Planets}

A second arena in which our understanding of astrophysical equations of state has recently undergone a considerable advance concerns the properties of dense $\mathrm{H}$ and in particular an improvement in our understanding of the manner in which the transition from neutral to ionized hydrogen occurs at low temperatures with increasing density ("pressure ionization"). There has been a long history of interest in the physics of this problem, and the astrophysical applications - especially to the interiors of the giant planets - have long been recognized. The problem of the equation of state of dense $H$ attracted renewed interest in the early 1980s with (1) the advent of experimentally attainable conditions approaching those needed for 
the pressure ionization (or equivalently the "metalization") of $\mathrm{H}$; (2) the theoretical predictions that pressure ionization may occur via a first-order "plasma phase transition" (PPT); and (3) the flurry of astrophysical interest in the properties of "brown dwarf" stars. It is beyond the scope of this paper to summarize all of the recent work on this problem, and I hope I may be forgiven for limiting myself to a few cases with which I am best acquainted.

Several groups have recently produced new equations of state for dense $H$ (see later papers in these proceedings). Among these, the calculations by Saumon (1989) and Saumon and Chabrier (1989) stand out in my opinion in that they have extended realistic EOS calculations to sufficiently high densities to encounter pressure ionization. They found, as had also been suggested by earlier, less detailed calculations (Robnik and Kundt 1983; Kraeft et al. 1986), that this transition appears to occur through a firstorder "plasma phase transition."

The theoretical prediction of the existence of the PPT by Saumon and Chabrier has not yet been confirmed experimentally, however. For this reason, there have been several recent efforts to find astrophysical tests of this result. In one effort, Chabrier, Saumon, and their colleagues have explored the consequences of the PPT for the structure and evolution of the giant planets and brown dwarfs (Chabrier et al. 1992; Saumon et al. 1992). While the effects they found were not large, they did discover that models including the first-order PPT were in slightly better agreement with the observational constraints than were models without the PPT.

A somewhat more promising test may involve studies of the effects of the PPT upon the oscillation modes of the giant planets. In the past several years, various groups have found observational evidence for periodic or quasiperiodic phenomena on Jupiter (Magalhães et al. 1989, 1990; Deming et al. 1989; Schmider and Mosser 1990; Schmider, Mosser, and Fossat 1991). Lee, Strohmayer, and Van Horn (1992) have recomputed the global oscillation modes of Jupiter taking account of the PPT. If it exists, the associated density discontinuity permits the existence of global oscillation modes trapped at the PPT; this may provide an observational test of the existence or non-existence of the PPT. In addition, Marley (1991) has also shown that if global oscillations are excited on Saturn, the ring system may be a very sensitive detector of these modes. 


\subsection{Nuclear Reaction Rates at High Densities}

Calculations of nuclear reaction rates at high densities are necessary for applications to supernova explosions and to accreting neutron stars and white dwarfs. Here I shall confine myself to brief discussions of three illustrative cases: the effects of screening on thermonuclear $\mathrm{C}+\mathrm{C}$ reactions in pre-supernova models, the extension of pycnonuclear (density-induced) reaction rate calculations to binary ionic mixtures (BIMs), and preliminary investigations of He-"burning" at zero temperature.

\subsubsection{C-ignition}

Calculations of the effects of electron-screening on the $\mathrm{C}+\mathrm{C}$ thermonuclear reaction rate date back to the original work by Salpeter (1954). He showed that the reaction rate is enhanced increasingly strongly by plasma screening at high densities. These calculations were subsequently improved by Salpeter and Van Horn (1969) and further refined by DeWitt, Graboske, and Cooper (1973). The statistical physics foundation for nuclear reactionrate calculations in dense plasmas was substantially clarified by Jancovici (1977) and Alastuey and Jancovici (1978).

Ichimaru (1993) has recently reviewed the calculation of nuclear reaction rates in very dense plasmas. Both thermonuclear and pycnonuclear rates at high densities depend upon evaluations of the screening potential at small separations of the reacting nuclei. While Monte Carlo calculations have now developed to the point where relatively accurate values can be obtained for the screening potential when two reacting nuclei are separated by distances comparable to the average distance between nuclei in the plasma, the extrapolation of these results to near-zero separation, where the nuclear reaction occurs, has recently become the subject of some controversy. Ogata, Iyetomi, and Ichimaru (1991) have employed their most recent, highly accurate Monte Carlo results, extrapolated to zero separation by a technique they developed, to obtain a parametrized form for the screened $\mathrm{C}+\mathrm{C}$ thermonuclear reaction rate both in the high-density fluid phase and in the solid phase of a $\mathrm{C} / \mathrm{O}$ plasma. This is the first detailed calculation of the enhancement factor for nuclear reaction rates for a binary ionic mixture (BIM). Ogata et al. found (i) that quantum-statistical effects greatly enhance the reaction rate above the value predicted by the classical enhancement factor and (ii) that the presence of $O$ in the solid phase acts to produce a blocking effect on the pycnonuclear reaction rates. The carbonignition curves are accordingly sensitive to the $\mathbf{O}$ abundance (cf. Ichimaru 
1993). The calculations by Ogata et al. have been criticized by Rosenfeld (1993), however, and the issue is currently unresolved.

\subsubsection{Pycnonuclear reactions for BIMs}

Ichimaru, Ogata, and Van Horn (1992) have developed an expression for the pycnonuclear reaction rate in a zero-temperature plasma which for the first time is valid for binary ionic mixtures (BIMs). Their result is:

$$
\begin{aligned}
P_{i j}= & \frac{1.34 \times 10^{32}}{1+\delta_{i j}} \frac{X_{i} X_{j}\left(A_{i}+A_{j}\right)}{Z_{i} Z_{j}\left(A_{i} A_{j}\right)^{2}} S_{i j} \rho^{2} \\
& \times \lambda_{i j}^{-1.809} \exp \left(-2.460 \lambda_{i j}^{-1 / 2}\right) \text { reactions } \mathrm{cm}^{-3} \mathrm{~s}^{-1}
\end{aligned}
$$

where $Z_{i}, A_{i}$, and $X_{i}(i=1,2)$ are, respectively, the atomic charges, masses, and mass fractions of the two reacting species. The quantities $S_{i j}$ are the cross-section factors (in MeV-barns). The dimensionless quantity $\lambda_{i j}$ is defined by

$$
\lambda_{i j}=\left(\frac{3}{4}\right)^{1 / 2} \frac{r_{i j}^{*}}{r_{\mathrm{m}, i j}},
$$

where the $r_{i j}^{*}=\left[\left(A_{i}+A_{j}\right) \hbar^{2}\right] / 2 A_{i} A_{j} H Z_{i} Z_{j} e^{2}$ are the nuclear Bohr radii, $a_{i j}=\frac{1}{2}\left(a_{i}+a_{j}\right)$ are the mean ion-sphere radii, and $r_{m, i j}$ are the observed peak positions of the pair correlation functions $g_{i j}(r)$. The energy generation rate $\varepsilon$ is given by $\varepsilon=P_{i j} Q / \rho$, where $Q$ is the energy released per reaction.

\subsubsection{He-"Burning" at $T=0$}

Pycnonuclear reaction rates for BIMs are necessary for calculations of the reactions subsequent to accretion onto the surfaces of neutron stars. The accepted model for X-ray burst sources is based on accretion of matter onto a neutron star from a low-mass, normal stellar companion. It is believed that hydrogen burns near the surface of the accreting neutron star at temperatures $\sim 10^{7} \mathrm{~K}$ and modest densities, leading to the accumulation of a $\sim 10^{7} \mathrm{~K}$ He layer of increasingly high density. When the density at the base of the accreted layer becomes great enough, He-burning is ignited, leading to a nuclear runaway. At sufficiently high accretion rates the temperature of the accreting matter is high, ignition involves screened thermonuclear reactions, and the resulting, relatively mild, event produces an X-ray burst (Joss 1978; Taam and Picklum 1979). At sufficiently low accretion rates, 
however, ignition may occur via pycnonuclear ractions and may be quite violent. Thus calculations of He-burning reactions at low temperatures are of considerable astrophysical interest.

Under normal astrophysical conditions, He burning occurs via the coupled reactions ${ }^{4} \mathrm{He}+{ }^{4} \mathrm{He} \rightarrow{ }^{8} \mathrm{Be}$ and ${ }^{4} \mathrm{He}+{ }^{8} \mathrm{Be} \rightarrow{ }^{12} \mathrm{C}^{*}$, which together comprise the so-called " $3 \alpha$ reaction." Both reactions are endoergic, and ${ }^{8} \mathrm{Be}$ and ${ }^{12} \mathrm{C}^{*}$ normally decay spontaneously into the original reactants. The ground state of ${ }^{12} \mathrm{C}$ is formed through a rare $\gamma$-decay of the excited state ${ }^{12} \mathrm{C}^{*}$. In the past few years, several authors have investigated the modifications of the $3 \alpha$ reaction that occurs with increasing density. Fushiki and Lamb (1987) showed how to take account of screening in these reactions by performing an $S$-matrix calculation for the $3 \alpha$ process. Their general calculation is valid for both the thermonuclear and pycnonuclear regimes. Among other things, they took into account the shift in the effective reaction energy produced by screening. More recently, Schramm, Langanke, and Koonin (1992) have done a $\mathrm{QM}$ calculation of the $3 \alpha$ reaction as a three-body problem, not as a succession of two-body problems.

An interesting alternative possibility is that He-burning may occur through a spontaneous, first-order phase transition instead of proceeding through the usual $3 \alpha$ reactions. When the density of the accumulated He at a neutron star surface increases to the point at which the electrostatic Coulomb interaction energy difference between a pair of reacting He nuclei and the resulting Be nucleus exceeds the $-92 \mathrm{keV}$ mass-energy difference, He ceases to be the lowest energy state. This occurs for the reaction ${ }^{4} \mathrm{He}+{ }^{4} \mathrm{He} \leftrightarrow{ }^{8} \mathrm{Be}$ when the condition for thermodynamic equilibrium, $2 \mu_{\mathrm{He}}-\mu_{\mathrm{Be}}=0$ is satisfied. Calculations show that this condition is achieved at a density $\rho=4.1 \times 10^{9} \mathrm{~g} \mathrm{~cm}^{-3}$ (Van Horn, Ogata, and Ichimaru 1991; Schramm, Langanke, and Koonin 1992 and references therein). This may have interesting consequences for the process by which matter accreted onto the surfaces of neutron stars reaches nuclear equilibrium at high densities, and thus affects the computations of the He shell-flashes that produce the X- or $\gamma$-ray bursts.

\subsection{Some Unsolved Problems}

In this final section, I conclude by merely listing a number of interesting problems that I do not have time to to discuss in detail but which I believe merit further study. 


\subsubsection{Matter in strong magnetic fields}

Since Ruderman's (1974) seminal paper on "magnetic matter," it has been recognized that the structure of matter in such strong magnetic fields at the surfaces of neutron stars is radically different from that of, e.g., the matter inside a white dwarf. Fushiki et al. (1989) have computed the equation of state in the non-relativistic limit, near the surface of a neutron star. In contrast with earlier calculations, they find that the density of matter does not approach zero gradually as the pressure $P$ falls to zero; instead they find a finite and large surface $(P=0)$ density, $\rho \sim$ several $\times 10^{3} \mathrm{~g} \mathrm{~cm}^{-3}$, for magnetic field strengths $\sim 10^{12}$ G. More recently, Lai and Shapiro (1991) have extended these calculations to include relativistic electrons. They have confirmed that the surfaces of strongly magnetic neutron stars have very large densities, increasing with the magnetic field strength. They have also studied the effects of such strong fields on the processes of $\beta$-decay and electron capture.

Two natural extensions of these calculations are (i) to augment them with new calculations of the transport properties of strongly magnetic matter and (ii) to incorporate the results into new models for the structure, evolution, and global oscillations of neutron stars. Some of these calculations are now beginning to be done ( $c f .$, Yakovlev 1993).

\subsubsection{Radiative opacities}

Another area in which substantial advances have occurred in recent years involves the calculation of radiative opacities at high densities. The OPAL project, led by F. J. Rogers and C. A. Iglesias at Lawrence Livermore National Laboratory (LLNL), showed that "non-hydrogenic" transitions (i.e., transitions in which the principal quantum number of the level remains unchanged) lead to a pronounced increase (more than a factor of two) in the opacity of typical astrophysical mixtures in the temperature range $T \sim 10^{5}$ to $10^{6} \mathrm{~K}$ (cf. Rogers, Iglesias, and Wilson 1987; Rogers and Iglesias 1992). The new OPAL opacities significantly improve the agreement both with astrophysical observations and with experimental data (Rogers and Iglesias 1992, 1993).

The second new initiative is the international Opacity Project, led by $M$. J. Seaton at University College London. This is a massive effort conducted by an international team of more than 25 scientists from at least seven nations to compute and compile the atomic data needed for astrophysical opacity calculations (cf. Seaton et al. 1992 and references therein). In contrast to the OPAL calculations, which use a single-configuration atomic 
model with configuration interactions to generate the atomic data needed for opacity calculations, the OP calculations focus instead on the computation of high-accuracy transition data, using sophisticated theoretical models for the physics of the atomic properties and processes. As a result of the massive database thus generated, and of the effort to validate the calculations against experimental data wherever possible, the OP effort is just beginning to produce astrophysically useful results. Where the OPAL and OP calculations can be compared, the results are now in very good agreement.

Another interesting, new opacity calculation was published recently by Lenzuni, Chernoff, and Salpeter (1991) for a gas consisting solely of $H$ and He, with no heavy elements. This calculation was motivated by an interest in the properties of the first generation of stars, which were formed prior to enrichment of the interstellar medium by the products of stellar nucleosynthesis. These calculations are also relevant to models for the atmospheres of very cool white dwarfs. One of the main contributions provided by these calculations is that the authors have fully included the effects of collisioninduced absorption by molecular hydrogen, which becomes the dominant process at high densities. Lenzuni et al. have incorporated the latest calculations of this process in their work.

Lenzuni and Saumon (1992) have subsequently extended these calculations to still higher densities, using the number densities from the equationof-state calculations by Saumon and Chabrier $(1989,1991)$. In addition to confirming the importance of collision-induced absorption by molecular $\mathrm{H}_{2}$, they found that absorption by $\mathrm{H}^{-}$plays an increasingly important role at the highest densities considered, as "pressure-dissociation and ionization" produces relative increases in the number densities of neutral $\mathrm{H}$ atoms and electrons. Only very approximate information is available concerning the properties of $\mathrm{H}^{-}$at very high densities, however, as Lenzuni and Saumon point out. Thus, the accuracy of the opacities at the very high densities remains uncertain, pending verification of the properties of $\mathrm{H}^{-}$in high-density plasmas.

Finally, it has been known for some time that plasma dispersion affects the absorption of radiation in dense plasmas (cf., Cox and Giuli 1968; Aharony and Opher 1979). However, this has never been taken into account properly in computations of astrophysical opacities. Because of the current interest in accurate, new calculations of opacities, Van Horn (1992) and Kurilenkov and Van Horn (1993) have recently initiated an effort to extend calculations of the Rosseland mean absorption coefficient $\kappa_{R}$ in a dense plasma to higher densities. They have obtained the following expression, which they are now 
working to extend to more realistic physical conditions:

$$
\frac{1}{\kappa_{R}}=\frac{\left.\int_{\omega_{p}}^{\infty} \frac{n_{\omega}^{3}}{\kappa_{\omega}^{(v a c)}} \frac{\partial B_{\omega}}{\partial T}\right|_{\omega} d \omega}{\left.\int_{0}^{\infty} \frac{\partial B_{\omega}}{\partial T}\right|_{\omega} d \omega}
$$

where the index of refraction of the plasma is $n_{\omega} \equiv(k c / \omega)=\left[1-\left(\omega_{p}^{2} / \omega^{2}\right)\right]^{l / 2}$, and the absorption coefficient in plasma has been taken to scale from the corresponding value in vacuum as $\kappa_{\omega}=n_{\omega}^{-1} \kappa_{\omega}^{(\text {vac })}$. It remains to incorporate this result into the current generation of astrophysical opacity calculations.

\section{Acknowledgements}

This work has been supported in part by NSF grant AST 91-15132 and NASA grant NAGW-2444 through the University of Rochester. In addition, I thank the organizers of this conference for providing me with the opportunity to atend, and I especially thank Gilles Chabrier for his patience in waiting for this much-delayed manuscript!

\section{References}

Aharony, U., and Opher, R., Astron. Ap., 79, (1979)

Alastuey, A., and Jancovici, B., $A p$. J., 226, 1034, (1978)

Barrat, J. L., Hansen, J. P., and Mochkovitch, R., Astr. Astr., 199, L15, (1988)

Blitz, L., Nature, 364, 757, (1993)

Chabrier, G., Saumon, D., Hubbard, W. B., and Lunine, J. I., Ap. J., 391, 817, (1992)

Clayton, D. D., Principles of Stellar Evolution and Nucleosynthesis (McGraw-Hill: New York), (1968)

Cox, J. P., and Giuli, R. T., Principles of Stellar Structure (Gordon and Breach: New York), pp. 64 ff., 189 ff, (1968)

Deming, D., Mumma, M. J., Espenak, F., Jennings, D. E., Kostiuk, T., Wiedemann, G., Lowenstein, R., and Piscitelli, J., Ap. J., 343, 456, (1989)

DeWitt, H. E., Graboske, H. C., Jr., and Cooper, M. C., Ap. J., 181, 439, (1973)

Fushiki, I., Gudmundsson, E. H., and Pethick, C. J., Ap. J., 342, 958, (1989)

Fushiki, I., and Lamb. D. Q., Ap. J., 317, 368, (1987)

Garcia-Berro, J., Hernanz, M., Mochkovitch, R., and Isern, J., Astron. Ap., 193, $141,(1988)$

Hernanz, M., Garcia-Berro, E., Isern, J., Mochkovitch, R., Segretain, L. and Chabrier, G., Ap.J, in press, (1994)

Iben, I., Jr., and Laughlin, G., Ap. J., 341, 312, (1989)

Ichimaru, S., Revs. Mod. Phys., 65, 255, (1993)

Ichimaru. S., Iyetomi, H., and Ogata, S., Ap. J., 334, L17, (1988)

Ichimaru, S., Ogata, S., and Van Horn, H. M., Ap. J., 401, L35, (1992)

Jancovici, B., J. Stat. Phys., 17, 357, (1977)

Joss, P., Ap. J., 225, L123, (1978)

Kraeft, W.-D., Kremp, D., Ebeling, W., and Röpke, G., Quantum Statistics of Charged Particle Systems (Plenum Press: New York), (1986) 
Kurilenkov, Yu. K., and Van Horn, H. M., these proceedings

Lee, U. M., Strohmayer, T. E., and Van Horn, H. M., Ap. J., 397, 674, (1992)

Lee, Y.-W., P. A. S. P., 104, 798, (1992)

Lenzuni, P., Chernoff, D., and Salpeter, E. E., Ap. J. Suppl., 76, 759, (1991)

Lenzuni, P., and Saumon, D., Rev. Mexicana Astron. Astrof., 23, 223, (1992)

Liebert, J., Dahn, C. C., and Monet, D. G., Ap. J., 332, 891, (1988)

Magalhães, J. A., Weir, A. L., Conrath, B. J., Gierasch, P. J., and Leroy, S. S., Nature, 337, 444, (1989)

Magalhães, J. A., Weir, A. L., Conrath, B. J., Gierasch, P. J., and Leroy, S. S., Icarus, 88, 39, (1990)

Marley, M. S., Icarus, 94, 420, (1991)

Norris, J. E., and Green, E. M., Ap. J., 337, 272, (1989)

Ogata, S., personal communication.

Ogata, S., Iyetomi, H., and Ichimaru, S., Ap. J., 372, 259, (1991)

Ogata, S., Iyetomi, H., Ichimaru, S., and Van Horn, H. M., Phys. Rev. E, 48, $1344,(1993)$

Robnik, M., and Kundt, W., Astron. Ap., 120, 227, (1983)

Rogers, F. J., and Iglesias, C. A., Ap. J. Suppl., 79, 507, (1992)

Rogers, F. J., and Iglesias, C. A., in Strongly Coupled Plasma Physics, ed. H. M.

Van Horn and S. Ichimaru (U. Rochester Press:Rochester, NY), p. 243, (1993)

Rogers, F. J., Iglesias, C. A., and Wilson, B. G., Ap. J., 322, L45, (1987)

Rosenfeld, $Y$., these proceedings

Ruderman, M. A., in IAU Symp. No. 59: Physics of Dense Matter, ed. C. J.

Hansen (Dordrecht: Reidel), p. 117, (1974)

Salpeter, E. E., Aust. J. Phys., 7, 373, (1954)

Salpeter, E. E., and Van Horn, H. M., Ap. J., 155, 183, (1969)

Sarajedini, A., and King, C. R., A. J., 98, 1624, (1989)

Saumon, D., Ph.D. thesis, University of Rochester, (1989)

Saumon, D., and Chabrier, G., Phys. Rev. Lett., 62, 2397, (1989)

Saumon, D., and Chabrier, G., Phys. Rev. A, 44, 5122, (1991)

Saumon, D., Hubbard, W. B., Chabrier, G., and Van Horn, H. M., Ap. J., 391, 827, (1992)

Schmider, F. X., and Mosser, G., in Progress of Seismology of the Sun and Stars, ed. Y. Osaki and H. Shibahashi (Springer: Berlin), (1990)

Schmider, F. X., Mosser, G., and Fossat, E., Astron. Ap., 248, 281, (1991)

Schramm, S., Langanke, K., and Koonin, S. E., Ap. J., 397, 579, (1992)

Seaton, M. J., Zeippen, C. J., Tully, J. A., Pradhan, A. K., Mendoza, C., Hibbert, A., and Berrington, K. A., Rev. Mexicana Astron. Astrof., 23, 19, (1992)

Segretain, L., and Chabrier, G., Astron. Ap., 271, L13, (1993)

Segretain, L., Chabrier, G., Hernanz, M., Garcia-Berro, E., Isern, J. and Mochkovitch, R., Ap.J, in press, (1994)

Stevenson, D. J., J. de Physique, Coll. C2, 41, C2-61, (1980)

Van Horn, H. M., Science, 252, 384, (1991)

Van Horn, H. M., Bull. A. A. S., 24, 824, (1992)

Van Horn, H. M., Ogata, S., and Ichimaru, S., Proc. US-Japan Workshop on Nuclear Fusion in Dense Plasmas, IFSR \#519, p. 57, (1991)

Winget, D. E., et al., Ap. J., 315, L77, (1987)

Yakovlev, D. G., in Strongly Coupled Plasma Physics, eds. H. M. Van Horn and S. Ichimaru (University of Rochester Press: Rochester, NY), (1993) 